\title{
Two diffusion photopolymer for sharp diffractive optical elements recording
}

\author{
S. Gallego, ${ }^{1, *}$ R. Fernández, ${ }^{1}$ A. Márquez, ${ }^{1}$ M. Ortuño, ${ }^{1}$ C. Neipp, ${ }^{1}$ M. R. Gleeson, ${ }^{2}$ \\ J. T. Sheridan, ${ }^{3}$ ANd A. Beléndez ${ }^{1}$ \\ ${ }^{1}$ Departamento de Física, Ingeniería de Sistemas y Teoría de la Señal, Universidad de Alicante, Apartado 99, E-03080 Alicante, Spain \\ 2III-V Materials and Devices, Photonics Center, Tyndall National Institute, Lee Maltings, Dyke Parade, Cork, Ireland \\ ${ }^{3}$ School of Electrical, Electronic and Communications Engineering, College of Engineering and Architecture, Communications and Optoelectronic \\ Research Centre, The SFI-Strategic Research Cluster in Solar Energy Conversion, University College Dublin, Belfield, Dublin 4, Ireland \\ *Corresponding author: sergi.gallego@ua.es
}

Received 21 April 2015; revised 29 May 2015; accepted 12 June 2015; posted 12 June 2015 (Doc. ID 238459); published 2 July 2015

Photopolymers as recording media are widely used in optical applications. In such materials, changes in the phase of the transmittance function are generated during exposure due to refractive index and thickness modulations. These changes arise primarily as a consequence of photopolymerization and mass transport processes. Characterizing polymers' performance, for example, quantifying the value of monomer diffusion, is therefore very important. Applying index matching, the volume and surface optical effect are separated in an acrylamide/polyvinylalcohol (AA/PVA) material. Using a simplified model that includes the effects of the holes produced during polymerization, both hole and monomer diffusion are analyzed. The analysis presented indicates higher material sensitivity than previously estimated. The results also indicate the possibility of recording sharper diffractive optical elements profiles, like blazed gratings, having diffraction efficiencies higher than 80\%. () 2015 Optical Society of America

OCIS codes: (160.5335) Photosensitive materials; (050.1950) Diffraction gratings; (160.2900) Optical storage materials.

http://dx.doi.org/10.1364/OL.40.003221

Because of their practical properties, self-processing photopolymers are frequently used in the mass production of holograms for security purposes and holographic-based memories [1]. Optical modulation of both the material permittivity and thickness can be achieved. Low scatter, inexpensive materials having wide spectral sensitivity are available. Material layers having a wide range of thicknesses can be obtained and their optical properties controlled. Thus photopolymers offer versatile media in which to fabricate surface relief diffractive-optical elements and volume holograms [2-4].

One such material, polyvinylalcohol/acrylamide (PVA/AA) [ $[-8]$, has received considerable attention. In these materials, grating formation occurs primarily as a consequence of photopolymerization and mass transport processes. However, a wide range of values for monomer diffusion have been reported in the literature, e.g., $10^{-7}$ to $10^{-10} \mathrm{~cm}^{2} / \mathrm{s}$ [5-8]. One result of assuming faster values, i.e., $10^{-7} \mathrm{~cm}^{2} / \mathrm{s}$, is that changes in the surface relief profile, due to mass transfer, make it difficult to produce sharp structures on the layer such as blazed or binary gratings, axicons, or diffractive lenses $[\underline{9}, \underline{10}]$.

In a recent study of PVA/AA [5], two sets of experimental data are analyzed: results involving the replay of coverplated and uncoverplated layers. In [5], coverplating was performed using silica oil to index match a glass plate. Based on the measured results for the coverplated samples rates of monomer, diffusion of at least $10^{-9} \mathrm{~cm}^{2} / \mathrm{s}$ and less than $10^{-11} \mathrm{~cm}^{2} / \mathrm{s}$ $[\underline{5,6]}$ were estimated. It is also mentioned as a hypothesis that heating during exposure may lead to some evaporation of water, and that post-exposure water molecules from the environment may diffuse into the layer [5].

Avoiding the exact origin of the surfaces changes, it is important to note that the presence of silica oil for index matching reduces the measured monomer diffusion, using some particular methods, more than factor 10 .

High values of monomer diffusion are useful for applications like volume holography, where the high spatial frequencies stored in the material permit the rapidly diffusing monomer molecules to move from the dark zones to bright ones where they are available to be polymerized. However, in applications like the recording of the diffractive lenses [2], high values of mass transport reduce the materials' ability to record sharp DOEs like lenses, blazed or binary gratings with enough quality [11].

Therefore, materials with low-matter transfer similar to photoresist can be used to record sharp diffractive optical elements with insignificant smoothing of the refractive index profiles [10].

In this Letter, glycerin is used to seal the PVA/AA material. A significant reduction in the estimated monomer diffusion is observed, and an improvement in the sharpness of the recorded DOEs is found. 
A simple model is developed to simulate the role of the sealant in the material, and the predictions of the model are compared to the experimental results. The model takes into account both the volume refractive index variations and the thickness variations. Holes are created as a consequence of the polymerization process in AA/PVA $[12,13]$. The holes, generated during the polymerization process, are the primary origin of the thickness variation. We assumed that the fast swelling of the illuminated areas (initially there is rapid shrinkage in the illuminated area), is due to the mass transport through the surface, clearly faster than the diffusion deep inside the material, and the surface tension forces.

The photopolymer formulation and experimental setup used for the experimental part are similar to those described previously, e.g., [8] using a $532 \mathrm{~nm}$ as the recording wavelength and a $633 \mathrm{~nm}$ as the readout one. In order to model the role of holes generation and holes diffusion, we propose the following model, where the grating vector is parallel to the $\mathrm{x}$-axis:

$$
\begin{aligned}
\frac{\partial M(x, z, t)}{\partial t}= & \frac{\partial}{\partial z} D_{m}(t) \frac{\partial M(x, z, t)}{\partial z}+\frac{\partial}{\partial x} D_{m}(t) \frac{\partial M(x, z, t)}{\partial x} \\
& -F_{R}(x, z, t) M(x, z, t) \\
& \frac{\partial P(x, z, t)}{\partial t}=F_{R}(x, z, t) M(x, z, t) \\
\frac{\partial H(x, t)}{\partial t}= & \frac{\partial}{\partial x} D_{h}(t) \frac{\partial H(x, t)}{\partial x}-K_{b}(x, t) M(x, t)
\end{aligned}
$$

where $M$ is the monomer volume fraction, $P$ is the polymer volume fraction, $H$ is the holes volume fraction, $F_{R}$ is the polymerization rate that depends on the dye concentration $[13,14]$, and is calculated as done previously assuming Beer's law [] $] K_{h}$, the holes rate generation $[\underline{12}, \underline{13}]$, is assumed proportional to $F_{R}$. $D_{m}$ and $D_{h}$ are the diffusion constant for holes and monomer, respectively. Holes are generated throughout the volume, and the resulting material shrinkage is most apparent by changes at the surface. It is reasonable to expect that holes diffusion will be much faster than that of the chemical compounds in the layer due to their small size.

In order to simulate the material behavior we assume that after shrinkage, the holes are concentrated close to the surface, and their motion is along $\mathrm{x}$ direction [see Eq. (3)].

$F_{R}, K_{h}, D_{h}$, and $D_{m}$ can be directly measured from zero spatial frequency and from behavior after exposure for very low spatial frequencies, without and with index matching, and also their variation with the polymer generation or time can be found [8]. Solving Eqs. (1)-(3) using finite difference method, the concentrations of the three components can be found as a function of time.

In order to quantify the phase of the DOE recorded, it is necessary to obtain the refractive and thickness variations as functions of the material concentrations. Regarding the index, the governing relation is

$$
\frac{n^{2}-1}{n^{2}+2}=\frac{n_{m}^{2}-1}{n_{m}^{2}+2} M+\frac{n_{p}^{2}-1}{n_{p}^{2}+2} P+\frac{n_{b}^{2}-1}{n_{b}^{2}+2}\left(1-M_{0}\right),
$$

where $M_{0}$ is the average initial value for the volume fraction of monomer, $n_{p}$ is the polymer refractive index, $n_{m}$ is the monomer refractive index, and $n_{b}$ is the binder refractive index. The local thickness variations in $\mathrm{x}$ are determined by the volume fraction of the holes:

$$
d(x)=d_{0}(x)-d_{h}(x)
$$

where $d$ is the thickness of the layer, $d_{0}$ is the intrinsic thickness (that can change due to monomer diffusion), and $d_{b}$ is the shrinkage due to the holes.

Using "zero frequency" interferometry, the values for monomer, polymer, and holes volumes can be calculated. If we assume that $d_{b}$, the thickness of the binder, is constant, we can obtain the resulting thickness of the layer. The initial value of $d$ for the layers presented in this Letter is $80 \pm 2 \mu \mathrm{m}$ (measured by ultrasound display provided by Neurtek). To obtain a good index matching and avoid the relief effects, we used a nonsoluble in-water liquid with refractive index 1.479 that fits perfectly with our layer before recording $(n=1.478)$ (measured by Abbe refractometer-WYA-1S). The phase change, $\Phi$, due to the surface relief pattern is

$$
\Phi(x)=n(x) d(x)+d_{h}(x) n_{h}
$$

and $n_{b}$ is 1 when the layers are not index matched, and 1.479 when we use the liquid. Throughout this Letter, we define the phase depth as the difference peak-to-peak of $\Phi$ along the x-axis and the phase modulation the amplitude.

Diffraction efficiencies (DE) for the main orders, measured in Fraunhofer region, of sinusoidal grating in transmission with a period $168 \mu \mathrm{m}$, and recording intensity of $0.5 \mathrm{~mW} / \mathrm{cm}^{2}$, both simulated and experimental, are depicted in Fig. 1(a) without index matching (no coverplating) and in Fig. 1(b) with index matching (coverplated). Due to the wavelength used, the refractive index modulation and the large period we are at
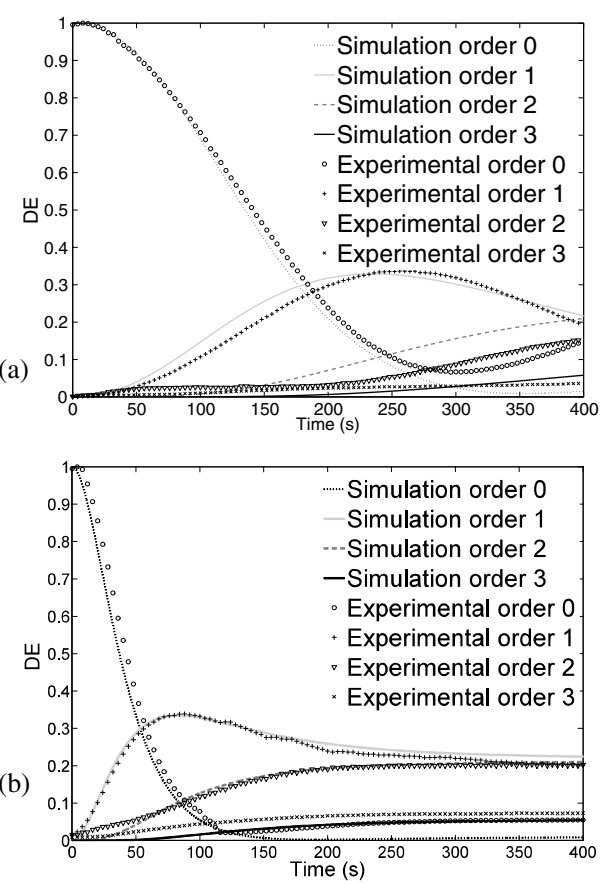

Fig. 1. DE of four main orders as a function of time. (a) Uncoverplated layers; (b) index matched (no surface changes effects). 
(a)

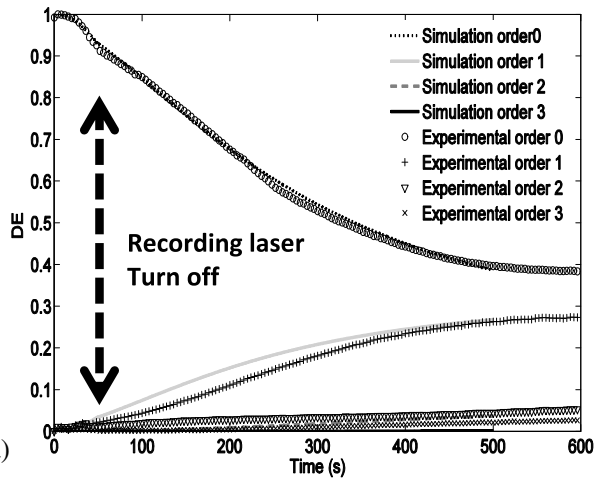

(b)

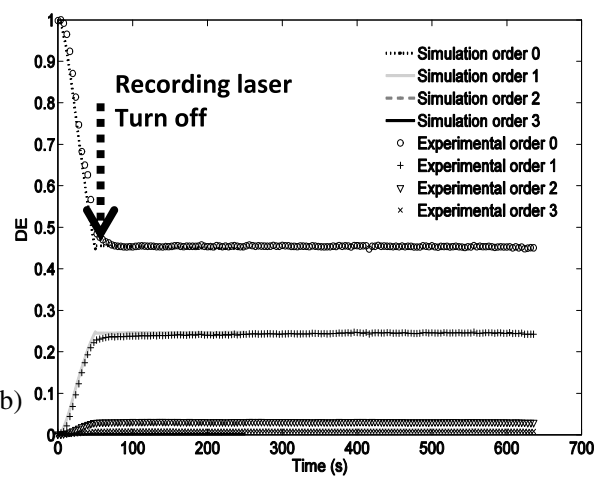

Fig. 2. DE of four main orders as a function of time for a $50 \mathrm{~s}$ of exposure and the post-evolution. (a) Uncoverplated layers; (b) coverplated and index matched (no surface effects).

Raman-Nath regime. As can be seen, the maximum values of the first order 1 (which is of primary interest for many diffractive applications) is achieved rapidly after only $80 \mathrm{~s}$ using the index-matching coverplated samples, in which the diffraction due to the effects of thickness variations are negligible. It is interesting to remark that in the coverplated case, the simulations and the experiments results are in a good agreement even after many minutes of recording.

To check the model capability to predict also the postexposure evolution in both cases, we recorded a grating during $50 \mathrm{~s}$, and then the recording laser was switched off. Our simulations are compared to the DE experimentally measured during the whole process during and after recording. As can be seen in Fig. 2(a), the DEs for the uncoverplated layers change significantly after recording due to the holes diffusion through the surface and their fast homogenization, using these data and following Ref. [8], $D_{h}$ can be calculated $\left(D_{h}=310^{-8} \mathrm{~cm}^{2} / \mathrm{s}\right)$. This result tells us that the surface changes, which are clearly visible in Fig. 2(a), are completely invisible optically in Fig. 2(b) due to the presence of the index matching. In other words volume effects stop rapidly [see Fig. 2(b)], but surface effects continue for a much longer time [see Fig. 2(a)]. Therefore, the hole diffusion parameter $D_{H}$ that is given is actually a lumped parameter quantifying the surface change.

When the index matching is used, Fig. 2(b), after recording, diffracted efficiencies rapidly stop to vary and their post exposure variation is slow. These changes are only due to the

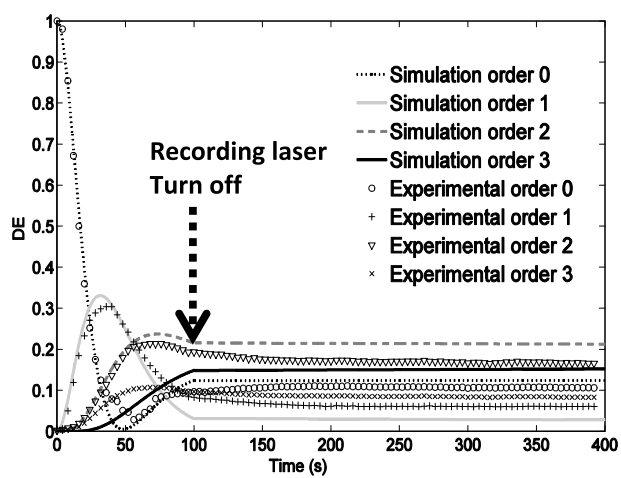

Fig. 3. DE of four main orders as a function of time for a $100 \mathrm{~s}$ of exposure and the post-evolution coverplated layer with BMA.

monomer diffusion inside the material. Thus, following the method described in [8], $D_{m}$ can be estimated $\left(D_{m}=\right.$ $10^{-10} \mathrm{~cm}^{2} / \mathrm{s}$ ) and introduced in the model to simulate the material behaviour. Due to the small value of $\mathrm{Dm}$, the evolution of the diffraction efficiencies after recording was monitored during $50 \mathrm{~min}$. Once both diffusions are obtained and introduced in the model, the simulations provided are very close to the reality as we show in Figs. $\underline{1}$ and 2 .

There are two possibilities to increase the depth of modulation of the surface profile: (i) maximize the thickness of the layer or (ii) achieve higher refractive index modulation by introducing new components like nanoparticles or cross-linkers [15]. In Fig. 3, the effect of incorporating the cross-linker, $\bar{N}, N^{\prime}$-methylene-bis-acrylamide (BMA), is shown. It can be seen that after $100 \mathrm{~s}$ of exposure, a phase modulation of $2 \pi$ is obtained [8].

This value of phase depth is enough to fabricate goodquality blazed gratings or diffractive lenses using this material thickness and composition. Figure $\underline{4}$ presents the simulations provided by the model using the typical parameters of composition with BMA compared with the experimental data. It is important to note the small discrepancies between experiments and simulations. They can be due to the low-pass filtering and the limited visibility of the optical system, which affects the recording pattern [16]. As it can be seen, the maximum of the order 1 is higher than $82 \%$ despite of the experimental limitations.

The results obtained in this Letter explain the discrepancies reported before by the measurements of monomer diffusion in PVA materials [5-7].

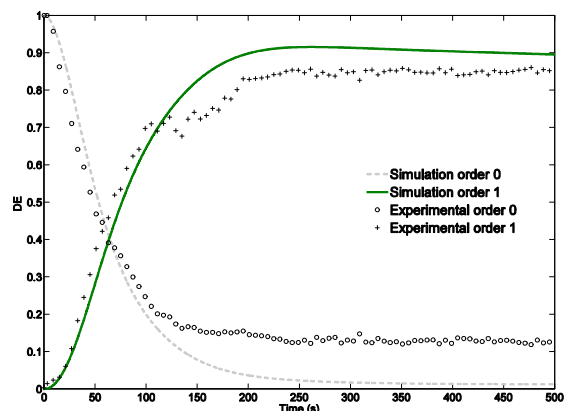

Fig. 4. Expected DE as a function of time to record blazed phase gratings into photopolymers. 
In addition, the values of the phase depth obtained for BMA cross-linker are much higher than those reported by other groups [5], achieving in this case enough phase depth to fabricate other diffractive elements such as lenses.

In addition, the dye elimination by curing process after the exposure can be carried out to the complete stabilization of the stored element.

In our opinion this provides a useful model to reproduce the experiments with and without index matching and try to close the polemical discussion with the ambiguous results reported about the acrylamide diffusion partially solved in [5]. This model is simple involving some approximations, but provides more physical information about the material than previous studies; the curves are not fitted using multiparametric models, and the simulations are obtained introducing the parameters measured directly. Then the model can be used as a tool to obtain the desired material or illumination parameters to fabricate a particular DOE, like Fig. 4 .

Funding. Enterprise Ireland; Generalitat Valenciana of Spain (ISIC/2012/013, PROMETEOII/2015/015); Irish Research Council for Science, Engineering and Technology (National Development Fund); Ministerio de Economía y Competitividad of Spain (FIS2011-29803-C02-01); Science Foundation Ireland.

Acknowledgment. J. T. S. and M. G. thank the IRC, SFI, and EI under the NDP.

\section{REFERENCES}

1. M. S. Weiser, F. K. Bruder, T. Fäcke, D. Hönel, D. Jurbergs, and T. Rölle, Macromol. Symp. 296, 133 (2010).

2. J. Neumann, K. S. Wieking, and D. Kip, Appl. Opt. 38, 5418 (1999).

3. F. T. O'Neill, A. J. Carr, S. M. Daniels, M. R. Gleeson, J. V. Kelly, J. R. Lawrence, and J. T. Sheridan, J. Mater. Sci. 40, 4129 (2005).

4. R. K. Kostuk, J. Castro, and D. Zhang, "Holographic low concentration ratio solar concentrators," in Frontiers in Optics, OSA Technical Digest (Optical Society of America, 2009), paper FMB3.

5. C. E. Close, M. R. Gleeson, and J. T. Sheridan, J. Opt. Soc. Am. B 28, 658 (2011).

6. C. E. Close, M. R. Gleeson, D. A. Mooney, and J. T. Sheridan, J. Opt. Soc. Am. B 28, 842 (2011).

7. T. Babeva, I. Naydenova, S. Martin, and V. Toal, Opt. Express 16, 8487 (2008).

8. S. Gallego, A. Marquez, F. J. Guardiola, M. Riquelme, R. Fernández, I. Pascual, and A. Beléndez, Opt. Express 21, 10995 (2013).

9. A. K. Kirby, P. J. Hands, and G. D. Love, Opt. Express 15, 13496 (2007).

10. P. Ruffieux, T. Scharf, I. Philipoussis, H. P. Herzig, R. Voelkel, and K. J. Weible, Opt. Express 16, 19541 (2008).

11. S. Gallego, A. Márquez, M. Ortuño, J. Francés, A. Beléndez, and I. Pascual, Opt. Express 20, 11218 (2012).

12. T. J. Bunning, L. V. Natarajan, V. P. Tondiglia, and R. L. Sutherland, Annu. Rev. Mater. Sci. 30, 83 (2000).

13. J. V. Kelly, M. R. Gleeson, C. Close, F. T. O'Neill, J. T. Sheridan, S. Gallego, and C. Neipp, J. Appl. Phys. 99, 113105 (2006).

14. G. M. Karpov, V. V. Obukhovsky, T. N. Smirnova, and V. V. Lemeshko, Opt. Commun. 174, 391 (2000).

15. E. Hata, K. Mitsube, K. Momose, and Y. Tomita, Opt. Mater. Express 1, 207 (2011).

16. S. Gallego, R. Fernández, A. Márquez, C. Neipp, A. Beléndez, and I. Pascual, Proc. SPIE 9131, 91311H (2014). 